\title{
Tunable kinematics of phase-slip lines in a superconducting stripe with magnetic dots
}

\author{
G. R. Berdiyorov, ${ }^{*}$ M. V. Milošević, and F. M. Peeters ${ }^{\dagger}$ \\ Departement Fysica, Universiteit Antwerpen, Groenenborgerlaan 171, B-2020 Antwerpen, Belgium \\ (Received 15 April 2009; revised manuscript received 13 October 2009; published 9 December 2009)
}

\begin{abstract}
Using numerical simulations, we study the dynamic properties of a superconducting stripe with a perpendicular magnetized ferromagnet on top in the presence of an applied dc current. In the "resistive" state conventional phase-slip lines are transformed into kinematic vortex-antivortex pairs with special dynamic behavior. In addition, the location of phase slippage in the sample is predetermined by the position of the magnetic dot. Both these effects directly influence the dynamics of the superconducting condensate and lead to periodic oscillations of the voltage across the sample with a frequency tunable both by the applied current and by the magnetization of the magnet. We found that the frequency of the voltage oscillations increases with increasing number of magnetic dots.
\end{abstract}

DOI: 10.1103/PhysRevB.80.214509 PACS number(s): 74.78. $-\mathrm{w}$, 73.23. $-\mathrm{b}, 74.40 .+\mathrm{k}$, 85.25. $-\mathrm{j}$

\section{INTRODUCTION}

Electric-field induced instabilities in current-carrying superconductors attracted a lot of attention since the discovery of the steplike features in the current-voltage characteristics of superconducting whiskers. ${ }^{1}$ Such an effect in onedimensional samples was nicely explained by phase-slip phenomenon (see Ref. 2 for review), according to which the phase of the order parameter periodically drops by $2 \pi$ in a single point. In such phase-slip centers (PSCs) the magnitude of the order parameter oscillates between zero and its maximum value. In two-dimensional (2D) superconductors the PSC may "grow" into a phase-slip line (PSL) ${ }^{3}$ (see also Ref. 4 for review), where the order parameter changes uniformly along one line perpendicular to the applied current. The oscillations of the order parameter in 2D samples are not necessarily constant along the PSL: they may occur in the form of propagating waves carrying the zero of the order parameter across the sample. Such waves (named kinematic vortices) have been discovered in numerical simulations using the 2D time-dependent Ginzburg-Landau (TDGL) equations, ${ }^{5}$ and experimental evidence for the existence of kinematic vortices was reported in Ref. 6. These kinematic vortices move with velocity $v_{k v} \approx 10^{5} \mathrm{~m} / \mathrm{s}$, which is much larger than the maximal measured speed of Abrikosov vortices ${ }^{7}$ $v_{A v} \approx 10^{3} \mathrm{~m} / \mathrm{s}$. Because of their very high velocity kinematic vortices do not retain their circular structure. ${ }^{5,8}$ The deformation of the vortex core, which originates from the finite relaxation time of the order parameter, ${ }^{9}$ leads to a rearrangement of the vortex lattice and a transition from slow to fast vortex motion ${ }^{10}$ compared to $v_{A v}$.

In order to avoid the dissipation in a superconducting system, due to the motion of vortices in the presence of transport current, artificial pinning centers are usually introduced, which can be arrays of microholes ${ }^{11,12}$ or magnetic dots. ${ }^{13,14}$ Vortex matter shows a rich variety of static and dynamic phases, which are well understood up to date (for review see Ref. 15). However, the behavior of kinematic vortices in the presence of a pinning landscape has not been studied yet.

In this paper we consider a superconducting stripe with magnetic dots on top (with out-of-plane moment) in applied dc current $I$. We show the repercussions of the presence of the magnets on the intrinsic properties of phase-slip lines, their dynamics and location, and the $I-V$ characteristics of the device. It is already known that in superconducting films with magnetic nanotextures the interaction of the vortices with each other and with the dot array gives rise to a rich variety of static and dynamic phases. ${ }^{13,16,17}$ For example, for sufficiently strong magnetization of out-of-plane magnetic dots, regular vortex-antivortex lattices are created ${ }^{16}$ (see also Ref. 18 for review) leading to a variety of commensurability effects and rich vortex-antivortex configurations. The critical properties of a superconductor can also be modified by the interaction with a nearby ferromagnet either through the electron exchange when in direct contact (i.e., proximity effect) or by the presence of the stray fields emanating from the ferromagnet (see, e.g., Refs. 13 and 17). In the latter case pinning effects can significantly enhance both the critical magnetic field and the critical current of the sample, especially at matching and fractional matching fields. ${ }^{13,17}$ Magnetic vortex ratchets have also been proposed in such superconducting/ferromagnet hybrids to control the vortex motion. ${ }^{19}$ Here we demonstrate that magnets can also be used to control the equilibrium dynamics of the system.

Oscillatory phenomena, which are an important part of the electronic scene, ${ }^{20}$ are readily found in nonequilibrium superconductivity, and many have been discussed in connection with Josephson junctions. ${ }^{21}$ However, singly-connected elements such as superconducting wires or stripes may also exhibit oscillatory features in the phase-slip regime. It was shown that ${ }^{5,8}$ dynamics of the phase-slip process leaves a clear mark on the electronic features of the sample: finite resistance but with resulting voltage along the sample with an ac added component with frequency determined by the relaxation of the superconducting condensate during phase slippage. Here we show that several aspects of the resistive state of a superconducting stripe can be manipulated by a nonhomogeneous magnetic field. For example, the resistive state of a superconducting stripe-the stability region of the resistive state, the frequency, and the amplitude of the voltage oscillations-is strongly influenced by the nonhomogeneous magnetic field of the dot.

The paper is organized as follows. In Sec. II we present our model system and theoretical approach. Section III is devoted to study the dynamics of kinematic vortex- 


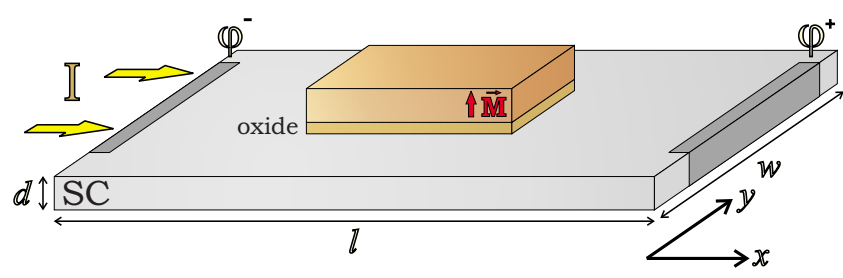

FIG. 1. (Color online) Oblique view of the device: superconducting stripe (size $w \times l \times d$ ) with a magnet on top (size $w_{m} \times l_{m}$ $\times d_{m}$ and magnetization $M$ ). Magnet and superconductor are electronically decoupled by an oxide of thickness $d_{o x}$. Given polarities of injected current $I$ and measured voltage $V=\varphi^{+}-\varphi^{-}$are denoted positive.

antivortex pairs in the presence of out-of-plane magnetized dot on top of the sample. Effect of several equidistant magnetic dots on the resistive state of the sample, as well as on the nucleation of vortex-antivortex pairs, is considered in Sec. IV. Our main findings are summarized in Sec. V.

\section{MODEL SYSTEM AND THEORETICAL APPROACH}

We consider a thin (thickness $d \ll \xi, \lambda$ ) superconducting strip (length $l$, width $w$ ) with a magnetic dot on top (size $\left.w_{m} \times l_{m} \times d_{m}\right)$ in the presence of perpendicular magnetic field $H$ and electric current applied through normal-metal leads [of size $b=w$ (see Fig. 1)]. We calculate the superconducting properties of the sample using TDGL theory, ${ }^{22}$ where we solve the 2D TDGL equation,

$$
\begin{gathered}
\frac{u}{\sqrt{1+\Gamma^{2}|\psi|^{2}}}\left(\frac{\partial}{\partial t}+i \varphi+\frac{\Gamma^{2}}{2} \frac{\partial|\psi|^{2}}{\partial t}\right) \psi \\
=(\nabla-i \mathbf{A})^{2} \psi+\left(1-|\psi|^{2}\right) \psi,
\end{gathered}
$$

coupled with the equation for the electrostatic potential $\Delta \varphi$ $=\operatorname{div}\left\{\operatorname{Im}\left[\Psi^{*}(\nabla-i \mathbf{A}) \Psi\right]\right\}$. We assume a thin superconductor with a good thermal contact with the substrate so that we do not have to couple the TDGL equations with the heat equation. Here, distances are scaled to coherence length $\xi$, time is in units of $\tau_{G L}=\pi \hbar / 8 k_{B} T u$, the electrostatic potential $\varphi$ is given in $\varphi_{0}=\hbar / 2 e \tau_{G L}$, and vector potential $\mathbf{A}$ is scaled by $H_{c 2} \xi$. The parameter $\Gamma=2 \tau_{E} \Psi_{0} / \hbar$ characterizes the chosen superconducting material (with $\tau_{E}$ being the inelastic electron-collision time and $\Psi_{0}$ is the value of the order parameter at zero temperature and no applied field). In the present simulations, we take $\xi=10 \mathrm{~nm}$ and $\lambda=200 \mathrm{~nm}$ at zero temperature (typical values for thin $\mathrm{Nb}$ films ${ }^{23}$ ), and remaining parameters are taken as $u=5.79$ and $\Gamma=20$. Using the normal-state resistivity $\rho=18.7 \mu \Omega \mathrm{cm}$ for such films we obtain $\tau_{G L} \approx 6.72$ ps. Neumann boundary conditions are taken at all sample boundaries, except at the current contacts where we used $\psi=0$ and $\left.\nabla \varphi\right|_{n}=-j_{\text {ext }}$ conditions, where $j_{\text {ext }}$ is applied current density. We discretized the equations using finite difference technique on a uniform 2D Cartesian grid (with grid spacing $0.1 \xi$ ) and used Euler iterative technique to solve the TDGL equation and Fourier transform method to solve the equation for the electrostatic potential.

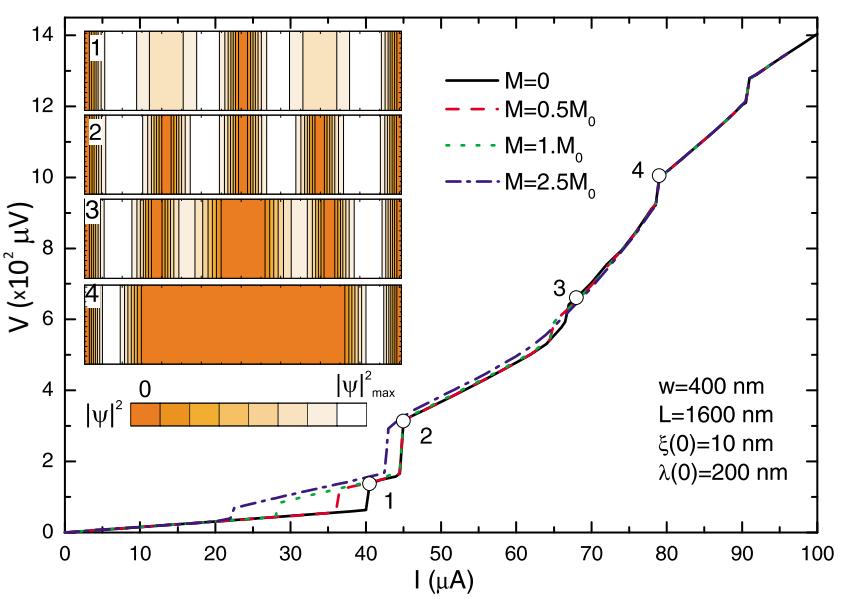

FIG. 2. (Color online) Current-voltage characteristics of the sample with dimensions $w=400 \mathrm{~nm}, l=1600 \mathrm{~nm}$, and $d=10 \mathrm{~nm}$ at $T=0.96 T_{c}$ for different magnetizations of the magnet (in units of $M_{0}=H_{c 2} / 4 \pi$ ). Cooper-pair density plots (the insets) show the distribution of phase-slip lines at different levels of dynamic resistance as indicated in the main panel for $M=0$.

\section{SINGLE MAGNETIC DOT: TRANSITION FROM PHASE-SLIP LINES TO KINEMATIC VORTEX- ANTIVORTEX PAIRS}

It is already well known that superconducting stripes go through several levels of increasing resistance as driving current is increased. 1,6,10,24 This is shown by solid black curve in Fig. 2 in a reference $I-V$ curve (for zero magnetization of the $\operatorname{dot} M=0$ and for the sample size $400 \times 1600 \mathrm{~nm}^{2}$ ). Zero resistance of the sample is maintained up to a threshold current (often denoted by $j_{c 2}$ ) when maximal current in the sample (at the middle of the sides) reaches the depairing current and causes phase-slippage and nonzero dynamic resistance $(\partial V / \partial I)$ of the sample (note that a small nonzero slope of the $I-V$ curve is due to the contact resistance from the leads). As a consequence, a phase-slip line nucleates in the center of the stripe, as shown in inset 1 of Fig. 2. Note that no kinematic vortices are observed for taken parameters of the stripe. With further increasing applied current, two more PSLs appear on each side of the central one, preserving the mirror symmetry (inset 2). Eventually, superconductivity becomes utterly suppressed in the center of the sample, leading to a state of higher resistance (inset 3 ); the process continues where the center PSL turns into a normal domain (inset 4) and finally toward complete destruction of superconductivity. In the following analysis, we place the magnetic dot of size $w_{m} \times l_{m} \times d_{m}=100 \times 200 \times 10 \mathrm{~nm}^{3}$ in the center of the superconducting stripe [vertically separated by an oxide layer of thickness $d_{o x}=10 \mathrm{~nm}$ (see Fig. 1)]. The vector potential of the stray magnetic field of the magnet in the superconducting plane is calculated numerically and scales with magnetization $M$ expressed in units of $M_{0}$ $=H_{c 2} / 4 \pi$. The presence of the magnetic dot leads to a larger current range of the "resistive" state as the phase-slip processes start to occur at lower applied current due to suppressed superconductivity under the magnet.

This is an important observation, as the focus of this study is the resistive state. Moreover, the magnetic dot has a pro- 

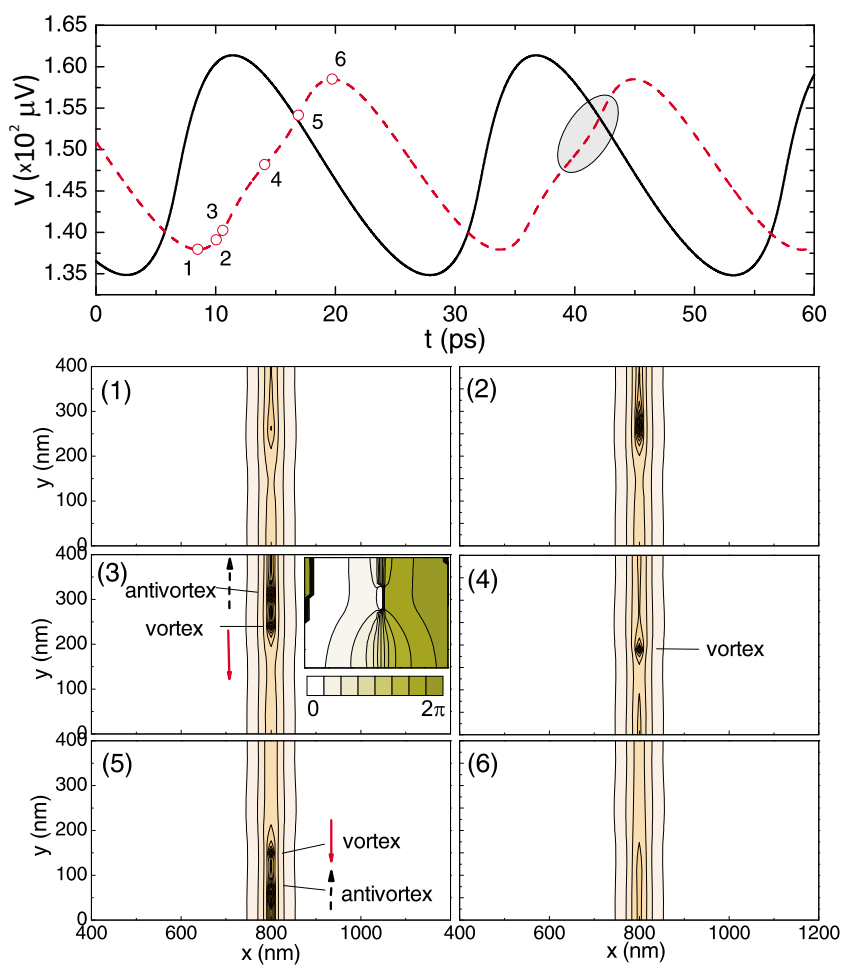

FIG. 3. (Color online) Equilibrated voltage vs time response of the sample at $I=42 \mu \mathrm{A}$ for $M=0$ (solid curve) and $M=0.4 M_{0}$ (dashed curve). The contour plots are snapshots of the Cooper-pair density at time intervals indicated in the main panel. Solid (dashed) arrow in (3) shows the direction of vortex (antivortex) motion. The inset of (3) shows the phase of the order parameter around the vortex-antivortex pair. Gray colored area in the main panel highlights the kink which is a consequence of the contraction of the phase-slip line into kinematic vortices.

found influence on the oscillations themselves: this is shown in Fig. 3, where the temporal voltage signal is compared for the cases with and without a magnetic dot on top of the stripe. For $M=0$, the observed oscillations are free from smaller features, and the order parameter changes uniformly across the sample. On the contrary, for $M>0$, we observed a kink in the voltage vs time characteristics (highlighted by a gray region in the main panel of Fig. 3), associated with a contraction of the phase-slip line into kinematic vortices. As shown by the contour plots of the Cooper-pair density in Fig. 3 , at the minimum of the measured voltage we observed the nucleation of a vortex-antivortex pair under the edge of the magnet (2). Under applied drive, antivortex moves toward the closest edge (3), and vortex moves in opposite direction. The vortex being in the center of the stripe, under the magnetic dot, presents a local energy minimum and results in a decreased voltage slope (note that due to the presence of the magnet, the total field piercing through the sample is positive but insufficient to stabilize the vortex there). Eventually, a new antivortex nucleates at the opposite edge of the sample (5) and meets the vortex where it annihilates under the edge of the magnet (6). This leads to maximal measured voltage, beyond which the superconducting condensate relaxes toward a new phase-slip process. In this way, the dynamics of the vortex is restricted only under the magnetic dot (4).

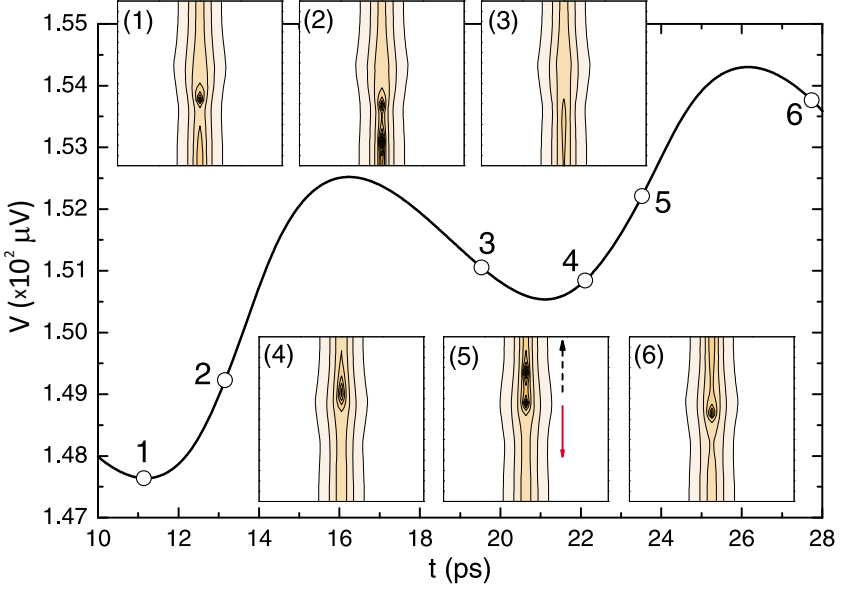

FIG. 4. (Color online) Equilibrated voltage vs time response of the sample at $I=42 \mu \mathrm{A}$ for $M=M_{0}$. Insets show snapshots of the Cooper-pair density. Solid (dashed) arrow in inset (5) shows the direction of vortex (antivortex) motion.

Obviously, the fine voltage features and relaxation time (and consequently voltage frequency) depend on the dot magnetization. The above discussed change in voltage slope becomes an actual dip for larger $M$, associated with stabilization of vortices under the magnetic dot. Figure 4 shows half the period of the voltage oscillation for $M=M_{0}$. For this value of the magnetization the global minimum corresponds to the state with one vortex pinned by the dot (inset 1). When time goes on an antivortex is created at the edge of the sample (2), which later annihilates with the pinned vortex. Vortex free state now corresponds to the local minimum of the voltage. Later, a vortex-antivortex pair nucleates at the top edge of the dot (inset 4). Antivortex leaves the sample (inset 5) and the vortex remains pinned inside the dot (inset $6)$, i.e., we arrive to the initial state (inset 1). Thus the dynamical process is opposite to the one shown in Fig. 3 for smaller magnetization of the dot.

In Fig. 5 we show the dependence of the amplitude and frequency of the voltage oscillations on the magnetization $M$ of the magnetic dot. We achieved a $37 \%$ increase of the frequency in the considered $M$ range (from 39.5 to over 54 $\mathrm{GHz}$ ) at driving current of $42 \mu \mathrm{A}$. Namely, increasing $M$ leads to increased screening currents circulating under the magnetic dot. The latter currents are opposing the applied current along one of the sample edges but enhancing it along the other edge. As a consequence, the increased $M$ leads to an increased gradient of the current across the sample, which speeds up the phase-slip dynamics. Simultaneously, the amplitude of the voltage oscillations decreases with magnetization. The process is continuous until the threshold $M$ for vortex stabilization is reached, beyond which we have a vortex present in the sample at each minimum of $V(t)$ curve. Therefore, the measurement $V(t)$ not only reveals the transformation of phase-slip lines into kinematic vortexantivortex pairs (see Fig. 3) but also provides means to detect vortex stabilization in the sample.

Finally, we study the effect of the off-center magnetic dot on the location and dynamics of the occurring phase slips. We shifted the magnet by $\Delta x=400 \mathrm{~nm}$ off center and calcu- 


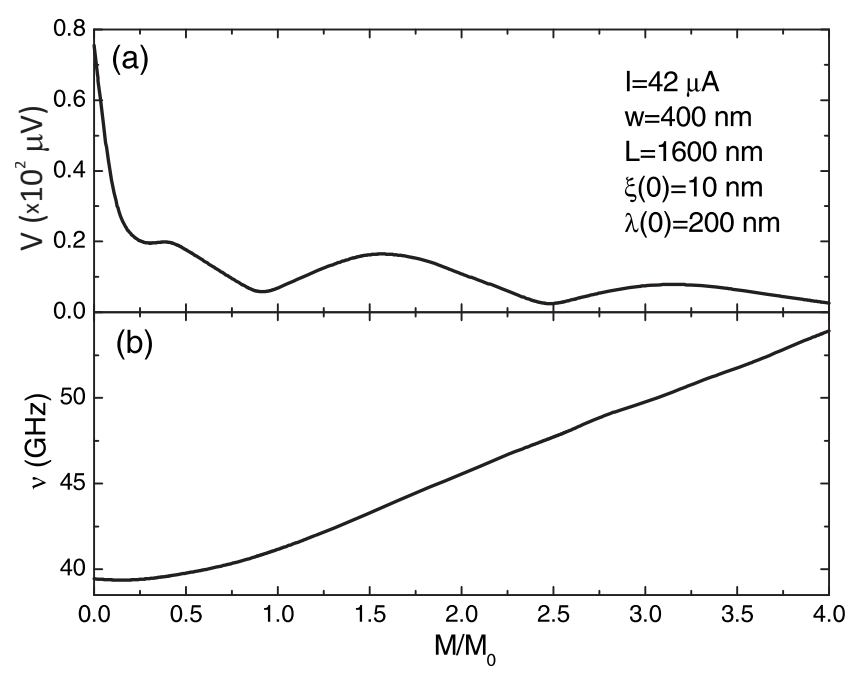

FIG. 5. (a) The amplitude and (b) the frequency of voltage oscillations as a function of magnetization $\mathbf{M}$ for $I=42 \mu \mathrm{A}$. While frequency is increasing with $M$, amplitude exhibits oscillations due to new vortex stabilized in the sample.

lated the $I-V$ characteristics shown in Fig. 6(a). As expected, the first phase slip now appears at the location of the magnetic dot (inset 1 in Fig. 6) and is transformed into vortexantivortex kinematic pairs as previously shown in Fig. 3 . However, differently from the case of the central magnet, the increased driving current leads to a state with two phase slips, one pinned under the dot and another at the center of the remainder of the stripe (inset 2). This leads to a new resistance level, not found in the case of a centered magnet. Further, two new phase slips occur on each side of the un-
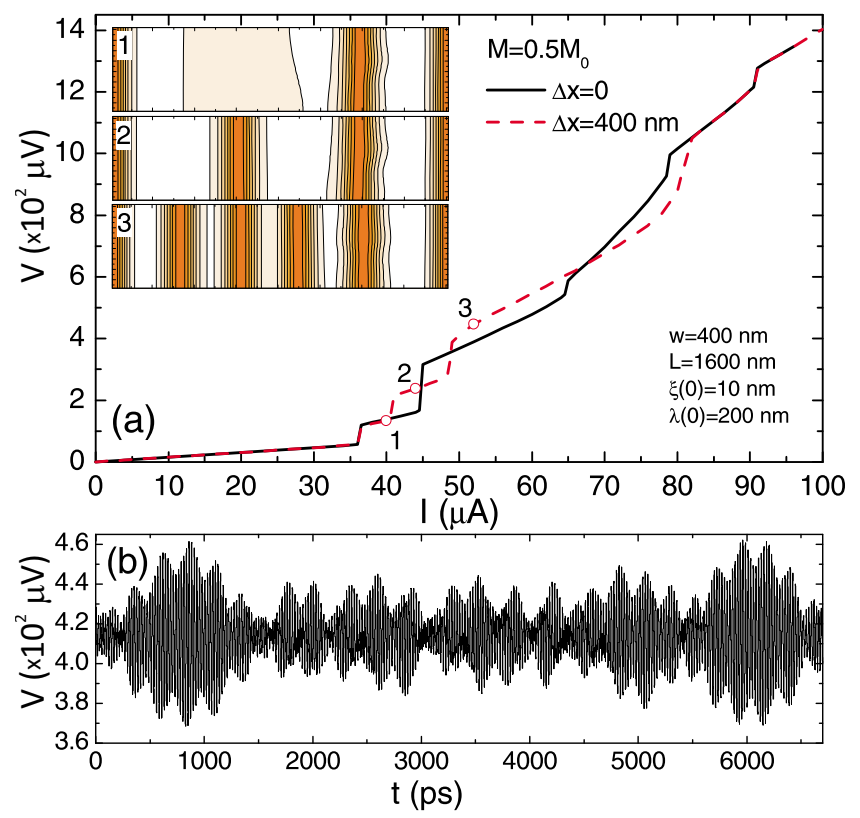

FIG. 6. (Color online) (a) $I-V$ characteristics and distribution of phase slips in the sample in Fig. 2 with an off-center magnet. The shift of the magnetic dot in $x$ direction is $\Delta x=400 \mathrm{~nm}$. (b) Multiharmonics voltage vs time in case when four phase slips are present in the sample [open circle 3 in (a)].

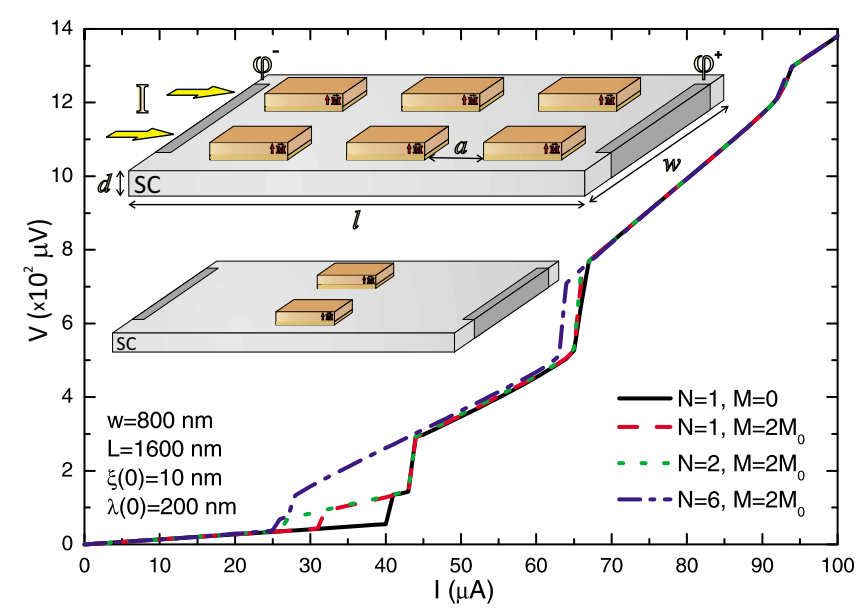

FIG. 7. (Color online) Current-voltage characteristics of a superconducting stripe with dimensions $w=800 \mathrm{~nm}, l=1600 \mathrm{~nm}$, and $d=10 \mathrm{~nm}$ at $T=0.96 T_{c}$ for one (red dashed curve), two (green dotted curve), and six (blue dashed-dotted curve) magnetic dots on top of the superconductor with magnetization $M=M_{0}$. The black solid curve shows the results when no magnetic dot is present. Insets show our model system - a superconducting stripe with two (bottom) and six (top) magnetic dots with size $w_{m} \times l_{m} \times d_{m}$ and separation $a$.

pinned phase-slip line (inset 3). This also provides for a new resistance level, corresponding to a total of four phase slips along the stripe. We conclude that an off-center position of the (small) magnetic dot (i) pins the first phase slip, (ii) effectively shortens the stripe for the nucleation of the following phase slips, and (iii) following from (ii) provides for resistance levels otherwise not found in a stripe with a centered dot. It should be noted here that the sequence of phase slips outside the dot region is the same as for the stripe without a dot (just on a shorter distance). However, the position of the magnetic dot breaks the symmetry of the current flow in the sample, as well as the symmetry of the positions of the phase-slip lines. As a consequence, all phase slips experience different current flows on their respective ends and oscillate out of phase. This leads to multiharmonic voltage oscillations shown in Fig. 6(b), which is due to the presence of four PSLs, which oscillates out of phase with its own frequency. The latter disregards the smaller features of each of the signals related to, e.g., phase-slip transformation under the magnet.

\section{MULTIPLE MAGNETIC DOTS: COEXISTENCE OF SLOW AND FAST MOVING VORTICES}

Up to now we studied the effect of a single magnetic dot on the dynamics of kinematic vortex-antivortex pairs in a finite size superconducting stripe. In what follows, we consider the case when several, equally spaced, magnetic dots are present on top of our sample. As an example, we consider a superconducting stripe with dimensions $w=800 \mathrm{~nm}, l$ $=1600 \mathrm{~nm}$, and $d=10 \mathrm{~nm}$ under two and six magnetic dots (see the insets in Fig. 7) with size $w_{m}=100 \mathrm{~nm}, l_{m}$ $=100 \mathrm{~nm}, d_{m}=10 \mathrm{~nm}$ and separation between them $a$ 
$=400 \mathrm{~nm}$. Here we present the results obtained for $T$ $=0.96 T_{c}$. We restrict ourselves to the case when the stray fields of the magnetic dots are weak, so that no vortexantivortex pairs are created at zero applied current. Figure 7 shows the $I-V$ curves of the sample with two (green dotted curve) and six (blue dashed-dotted curve) magnetic dots with magnetization $M=2 M_{0}$. As a reference curve we also plotted the results for the sample without (black solid curve) and with a single (red dashed curve) magnetic dot in the middle of the sample. The presence of magnetic dots does not qualitatively change the general behavior of the current-voltage characteristics of the sample, i.e., the stepwise increase of the average voltage is found as a function of applied current. In comparison with the reference curve, samples with magnetic dots exhibit the resistive state in a larger current range as phase slippage occurs at lower threshold currents. The resistive state in the latter case is caused by periodic generation of kinematic vortex-antivortex pairs which leads to a nonzero time-averaged resistance. With increasing the number of magnetic dots the critical current for the transition from the fully superconducting state to the resistive state becomes smaller due to the suppression of the order parameter under the magnetic dots. However, critical current for superconducting (resistive)/normal transition does not depend on the number of magnetic dots.

To see the effect of closely located magnetic dots on the dynamics of kinematic vortices, we plotted in Fig. 8 the voltage oscillations when two magnetic dots are present with magnetization $M=2 M_{0}$ (blue dotted curves) for the applied current $I=42 \mu \mathrm{A}$. For comparison, we also show the temporal voltage signal without (black solid curve) and with one (red dashed curve) magnetic dot, which is located in the middle of the sample. As we see from these $V(t)$ curves, the period of the voltage oscillations slightly decreases (i.e., the frequency of the oscillations increases) with increasing the number of magnetic dots. The dip in the $V(t)$ curve in the case of one magnetic dot, which we have related to the stabilization of a vortex under the dot (see the discussion of Fig. 4 ), is not pronounced in the case of two magnetic dots. The latter is due to the different dynamics of the kinematic vortices, as illustrated by the contour plots of the phase of the order parameter in Fig. 8. For these values of the magnetization and the applied drive, one vortex is pinned by the bottom dot when the $V(t)$ curve has a minimum (see inset 1). Afterward, a vortex-antivortex pair nucleates under the edge of the top (vortex free) magnetic dot close to the edge of the superconducting stripe (inset 2), where the supercurrent distribution is maximal. Under the effect of the Lorentz force the antivortex leaves the sample toward the top boundary. At the same time a new antivortex penetrates the sample from the opposite edge of the sample and annihilates with the vortex pinned under the dot (inset 2). Thus, we again have only one vortex now pinned by the other magnetic dot. Later a new pair of vortices with opposite polarization is created under the edge (closest to the neighboring dot) of the bottom dot (inset 4). The antivortex moves toward the pinned vortex by the top magnetic dot (inset 5) and annihilates with it. Consequently, the system arrives back to the initial state (inset 6). This is the complete cycle of vortex-antivortex creation or annihilation, responsible for the voltage oscillations in the sample.
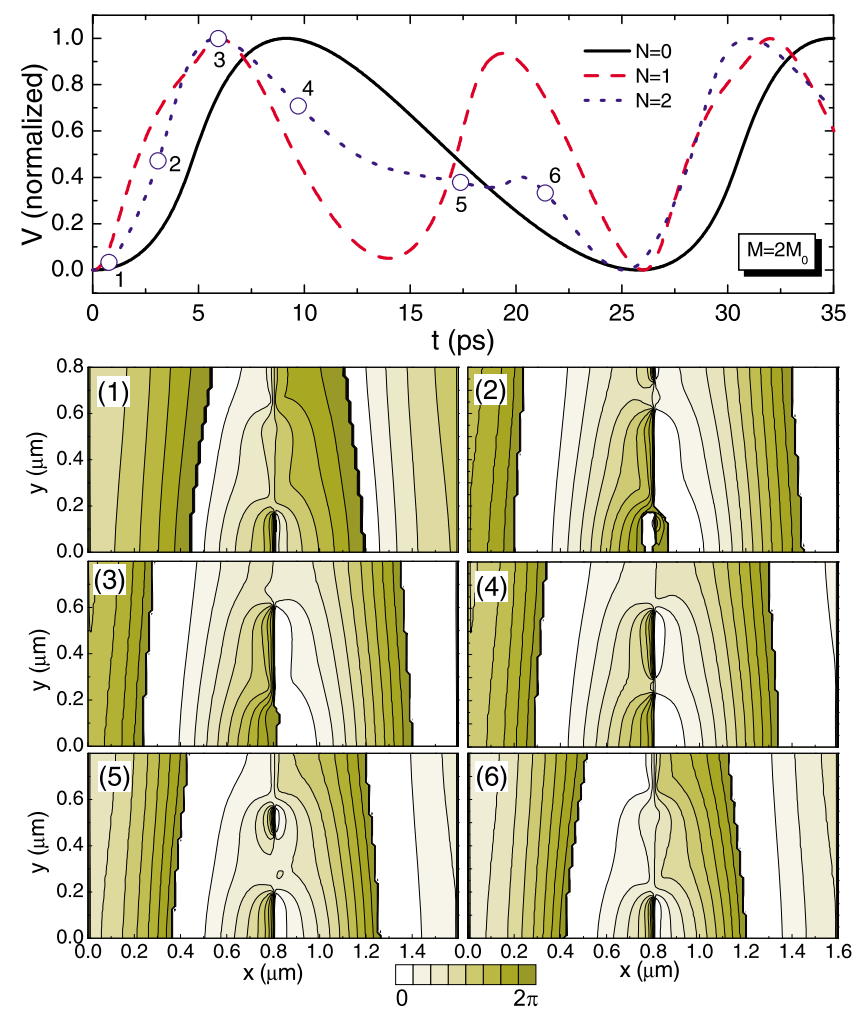

FIG. 8. (Color online) Equilibrated voltage vs time response of the sample in Fig. 7 without (black solid curve) and with one (red dashed curve) and two (blue dotted curve) magnetic dots of magnetization $M=2 M_{0}$ at $I=42 \mu \mathrm{A}$. The contour plots are snapshots of the phase of the order parameter at time intervals indicated in the main panel by open circles for the sample with two magnetic dots.

Figure 9 shows the voltage vs time response of the sample with six magnetic dots with magnetization $M=2 M_{0}$ for applied current $I=26 \mu \mathrm{A}$. At this value of the applied drive the system is in the resistive state with one phase-slip line in the middle of the sample. Moreover, the magnetic moments of the dots are large enough to create a vortex-antivortex pair away from the middle of the sample, which moves under the action of the Lorentz force. As the behavior of kinematic vortices in the vortex channel is similar to the behavior of those when only two central magnetic dots are present (see the discussion of Fig. 8), we only focus here on the dynamics of the slow moving Abrikosov vortices generated by the offcenter magnetic dots. Due to the nonlinear distribution of the currents in our finite sample, first the upper dots generate a vortex and antivortex pair near the edge of the sample (inset 1). The antivortex leaves the sample and the vortex remains pinned under the dot due to the positive magnetic field (inset 2 ). Note that such a single vortex per magnetic dot cannot be generated in superconducting films with magnetic dot arrays due to phase conservation (total flux equals zero). Later, another pair nucleates under the bottom dots (inset 4) with an antivortex annihilating with the vortex pinned under the top dot (inset 5). With time going on, an antivortex penetrates the sample through the bottom edge of the sample and annihilates with the vortices under the bottom dots (not shown here). Thus, the dynamics of Abrikosov vortex-antivortex pairs is similar to the dynamics of kinematic vortices. How- 

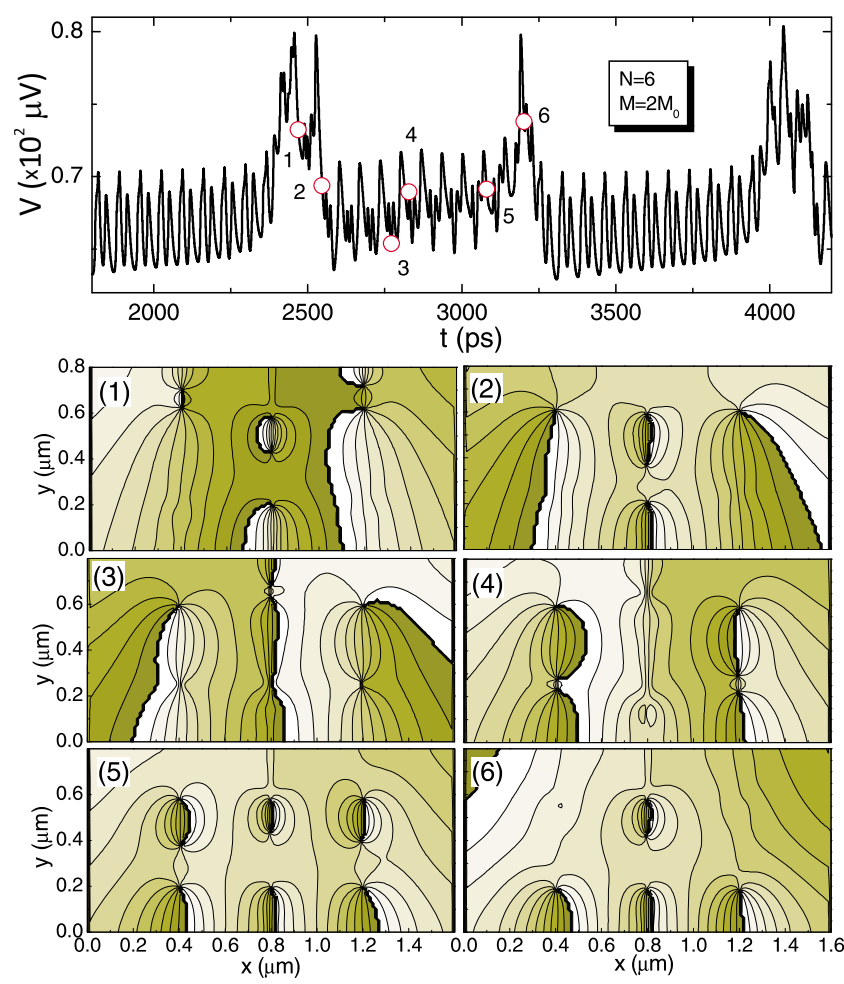

FIG. 9. (Color online) $V(t)$ curve of the sample with six magnetic dots with magnetization $M=2 M_{0}$. The applied current is $I$ $=26 \mu \mathrm{A}$, which generates one phase-slip line in the middle of the sample. The contour plots are snapshots of the phase of the order parameter at time intervals indicated in the main panel.

ever, creation and/or annihilation processes of Abrikosov vortices are far slower that the ones for kinematic vortices, leading to significant maxima in the $V(t)$ curve. Fast moving kinematic vortices lead to high frequency oscillations be- tween these maxima. Thus, we have a coexistence of fast and slow moving vortices. ${ }^{10}$

\section{CONCLUSIONS}

In conclusion, we analyzed the properties of the resistive state in a superconducting stripe with magnetic dots on top. Magnetic dot with perpendicular magnetization was found to increase the range of applied current in which the resistive state is realized. Further, it leads to the formation of a kinematic vortex-antivortex pair with very distinct dynamic behavior. The evidence for vortex-antivortex nucleation and annihilation can be found in particular features of the voltage vs time signal. The latter signal also proves the possibility for tuning the frequency of the oscillations by the magnetization of the dot and contains information about vortex stabilization in the sample - the appearance of vortices in the sample can be detected through discontinuities in the amplitude vs magnetization curve. We found that a magnetic dot is a very effective phase-slip pinning center, with the consequence that it may break the symmetry of the resistive state, it can lead to different levels of resistance compared to the symmetric case and can generate a complex multiharmonic output voltage.

We also considered the case when several equally spaced magnetic dots are present on top of the superconducting stripe. We found that with increasing number of dots the frequency of the voltage oscillations increases. The coexistence of slow (Abrikosov) and fast (kinematic) moving vortices are found, which exhibit similar dynamic behavior, but leaves different traces on the voltage vs time characteristics.

\section{ACKNOWLEDGMENTS}

This work was supported by the Flemish Science Foundation (FWO-Vl) and the Belgian Science Policy (IAP). G.R.B. acknowledges support from FWO-Vlaanderen. *golibjon.berdiyorov@ua.ac.be

†rancois.peeters@ua.ac.be

${ }^{1}$ J. Meyer and G. v. Minnigerode, Phys. Lett. 38A, 529 (1972).

${ }^{2}$ B. I. Ivlev and N. B. Kopnin, Usp. Fiz. Nauk 142, 435 (1984) [Sov. Phys. Usp. 27, 206 (1984)].

${ }^{3}$ A. Weber and L. Kramer, J. Low Temp. Phys. 84, 289 (1991).

${ }^{4}$ I. M. Dmitrenko, Fiz. Nizk. Temp. 22, 849 (1996) [J. Low Temp. Phys. 22, 648 (1996)].

${ }^{5}$ A. Andronov, I. Gordion, V. Kurin, I. Nefedov, and I. Shereshevsky, Physica C 213, 193 (1993).

${ }^{6}$ A. G. Sivakov, A. M. Glukhov, A. N. Omelyanchouk, Y. Koval, P. Müller, and A. V. Ustinov, Phys. Rev. Lett. 91, 267001 (2003).

${ }^{7}$ M. Tinkham, J. Low Temp. Phys. 35, 147 (1979).

${ }^{8}$ G. R. Berdiyorov, M. V. Milošević, and F. M. Peeters, Phys. Rev. B 79, 184506 (2009).

${ }^{9}$ L. I. Glazman, Fiz. Nizk. Temp. 12, 688 (1986) [Sov. J. Low Temp. Phys. 12, 389 (1986)].

${ }^{10}$ D. Y. Vodolazov and F. M. Peeters, Phys. Rev. B 76, 014521
(2007)

${ }^{11}$ A. T. Fiory, A. F. Hebard, and S. Somekh, Appl. Phys. Lett. 32, 73 (1978)

${ }^{12}$ M. Baert, V. V. Metlushko, R. Jonckheere, V. V. Moshchalkov, and Y. Bruynseraede, Phys. Rev. Lett. 74, 3269 (1995).

${ }^{13}$ J. I. Martin, M. Vélez, J. Nogués, and I. K. Schuller, Phys. Rev. Lett. 79, 1929 (1997).

${ }^{14}$ M. J. Van Bael, M. Lange, S. Raedts, V. V. Moshchalkov, A. N. Grigorenko, and S. J. Bending, Phys. Rev. B 68, 014509 (2003); M. V. Milošević, S. V. Yampolskii, and F. M. Peeters, ibid. 66, 174519 (2002).

${ }^{15}$ G. Blatter, M. V. Feigel'man, V. B. Geshkenbein, A. I. Larkin, and V. M. Vinokur, Rev. Mod. Phys. 66, 1125 (1994); G. W. Crabtree and D. R. Nelson, Phys. Today 50(4), 38 (1997).

${ }^{16}$ M. V. Milošević and F. M. Peeters, Phys. Rev. B 68, 094510 (2003); Phys. Rev. Lett. 93, 267006 (2004); 94, 227001 (2005); Europhys. Lett. 70, 670 (2005).

${ }^{17}$ M. V. Milošević, G. R. Berdiyorov, and F. M. Peeters, Phys. Rev. Lett. 95, 147004 (2005). 
${ }^{18}$ M. Velez, J. I. Martín, J. E. Villegas, A. Hoffmann, E. M. González, J. L. Vicent, and I. K. Schuller, J. Magn. Magn. Mater. 320, 2547 (2008).

${ }^{19}$ G. Carneiro, Physica C 432, 206 (2005).

${ }^{20} \mathrm{M}$. H. Jones, A Practical Introduction to Electronic Circuits (Cambridge University Press, Cambridge, 1982).

${ }^{21}$ K. K. Likharev, Dynamics of Josephson Junctions and Circuits (Gordon and Breach Science, Amsterdam, 1986).

${ }^{22}$ L. Kramer and R. J. Watts-Tobin, Phys. Rev. Lett. 40, 1041
(1978); R. J. Watts-Tobin, Y. Krähenbühl, and L. Kramer, J. Low Temp. Phys. 42, 459 (1981).

${ }^{23}$ A. I. Gubin, K. S. Il'in, S. A. Vitusevich, M. Siegel, and N. Klein, Phys. Rev. B 72, 064503 (2005).

${ }^{24}$ D. Y. Vodolazov, F. M. Peeters, L. Piraux, S. Mátéfi-Tempfli, and S. Michotte, Phys. Rev. Lett. 91, 157001 (2003); A. K. Elmurodov, F. M. Peeters, D. Y. Vodolazov, S. Michotte, S. Adam, F. de Menten de Horne, L. Piraux, D. Lucot, and D. Mailly, Phys. Rev. B 78, 214519 (2008). 